\title{
Reduction of Wear Volume from Accelerated Aged UHMWPE Knee Components by the Addition of Vitamin E*
}

\author{
Satoshi TERAMURA**, Hideyuki SAKODA***, Tomohiro TERAO****, \\ Kunihiko FUJIWARA***, Kosuke KAWAI** and Naohide TOMITA** \\ ** Graduate School of Engineering, Kyoto University, \\ Yoshida-Honmachi, Sakyo-ku, Kyoto, Kyoto 606-8501, Japan \\ E-mail: ntomita@iic.kyoto-u.ac.jp \\ *** Development department, Nakashima Medical Co., Ltd., \\ Joto-Kitagata, Higashi-ku, Okayama, Okayama 709-0625, Japan \\ **** Graduate School of Medicine, Kyoto University, \\ Yoshida-Konoe-Chou, Sakyo-ku, Kyoto, Kyoto 606-8501, Japan
}

\begin{abstract}
Accelerated ageing was conducted on UHMWPE with and without vitamin E (D, $\mathrm{L}-\alpha$ tocopherol, VE). Wear performance was investigated using a knee simulator and wear debris was analysed. Aged UHMWPE with VE showed significantly lower wear volume than that of aged virgin UHMWPE and showed approximately similar wear volume as non-aged virgin UHMWPE. There were no significant differences among the materials as far as shape factor of the debris is concerned.
\end{abstract}

Key words: Medical Engineering, Biomaterials, Material Testing, Wear, Bearing, UHMWPE, Vitamin E, Artificial Joint, Wear Debris, Accelerated Ageing

\section{Introduction}

Ultra high molecular weight polyethylene (UHMWPE) has been the most successful material for artificial joints and is widely used as bearing material. However the longevity of an artificial joint consisting of UHMWPE was limited due to wear and wear related problems such as osteolysis and loosening.

UHMWPE containing vitamin E (D, L- $\alpha$ tocopherol, VE) addition has been gaining much attention as an implant material for artificial hip and knee joints. Sample preparation procedure for UHMWPE containing VE has been fallen into two general categories, blend and dope method. As VE-blended UHMWPE, It has been reported that reduction in wear volume and sub-micron sized debris using a knee simulator which suggests less impact to osteolysis $^{(1)}$, lower wear volume using pin-on-disc testing ${ }^{(2)}$, lower percentage of internal defects and area of cracks ${ }^{(3)}$, improvement in fatigue strength ${ }^{(2)}$, preventing increased surface roughness during bi-directional sliding fatigue test ${ }^{(4)}$, no noteworthy oxidative degradation and difference with conventional UHMWPE in animal experiment ${ }^{(5)}$, also lower oxidative degradation before ${ }^{(6)(7)}$ and even after various accelerated ageing treatments ${ }^{(8)-(10)}$, no toxic effects on human cells in vitro ${ }^{(11)}$, a reduced amount of fragments and single heavy chain $\operatorname{IgG}$ adsorption making it less prone to foreign-body reaction ${ }^{(12)}$, and less crosslink density (13)-(14), than that of conventional UHMWPE respectively. Also as VE-doped UHMWPE, similar effects such as lower oxidation degree after various accelerated ageing treatments ${ }^{(15)}$, lower wear volume using a hip simulator with and without third body bone cement ${ }^{(16)}$ as well as a pin-on-disc testing ${ }^{(16)}$, than that of conventional UHMWPE. These results suggested that UHMWPE containing vitamin E

*Received 28 Aug., 2009 (No. 09-0472) [DOI: 10.1299/jbse.4.589] 
provided a greater longevity when used as an artificial joint component. However, the positive effects should be confirmed even after the accelerated ageing treatment.

In the current study therefore, a knee simulator test was carried out on aged UHMWPE with and without VE to validate our assumption that VE addition has positive effects on UHMWPE after severely aged conditions. Shape of wear debris was analysed to estimate the influence of the debris on osteolysis.

\section{Materials and Methods}

\section{Materials}

Accelerated aged virgin and $0.3 \mathrm{wt} \%$ VE-added UHMWPE were prepared in the current study. Virgin UHMWPE was manufactured using a direct compression moulding technique at $220{ }^{\circ} \mathrm{C}$ under $25 \mathrm{MPa}$ for 30 minutes from UHMWPE GUR1050 resin powder (Ticona, USA). For UHMWPE containing VE, the powder was mixed with VE (DL- $\alpha$ Tocopherol JP, Eisai, Japan) at $0.3 \mathrm{wt} \%$ using a screw cone mixer (High Speed Mixer Type, LFS-GS-2J, Fukae Powtec, Japan). After mixing, the tibial component of the knee joint was made from the mixed powder under the same conditions as the virgin UHMWPE. After the consolidation, $\gamma$-Irradiation was conducted in air $\left(\mathrm{Co}^{60}\right)$ with the smallest dose of $2.68 \mathrm{Mrad}$. After the irradiation, both UHMWPEs were exposed to room temperature in air for 7 days then were subjected to ageing in a convection oven (DN400, YAMATO SCIENTIFIC CO.,LTD, Japan). The ageing condition $\left(80^{\circ} \mathrm{C}\right.$ for 23 days in air) has been indicated to be equivalent to 5-10 years of natural ageing in air-irradiated UHMWPE ${ }^{(17)(18)}$.

Knee simulator test

A six-station four-axis knee joint simulator (AMTI-Boston 6 Station Knee Simulator, AMTI, USA) was used for the knee simulator test (Fig. 1). The maximum load was $2600 \mathrm{~N}$, flexion angle was $58^{\circ}$, internal external rotation was from -5.68 to $1.75^{\circ}$ and anterior posterior translation was from 0 to $5.15^{\circ}$. Hi-tech Knee II Total Knee System (NAKASHIMA Medical, Japan) constructed of Ti-6Al-4V alloy and UHMWPE was used as femoral component. A single lot of bovine calf serum (JRH BIOSCIENCES, Inc., USA) diluted to $25 \%$ with $0.3 \%$ sodium azide (Wako Pure Chemical Industries, Ltd., Japan) was used as a lubricant. The lubricant was changed every 0.5 million cycles and the test lasted for five million cycles. Gravimetric wear was measured (Mettler Toledo AX205 Delta Range, Mettler-Toledo $\mathrm{GmbH}$, Switzerland) at the first 0.5 million cycles and every one million cycles thereafter. The specimens were weighed to the accuracy of $10 \mu \mathrm{g}$ together with weight control specimens in order to minimize the effect of water uptake.

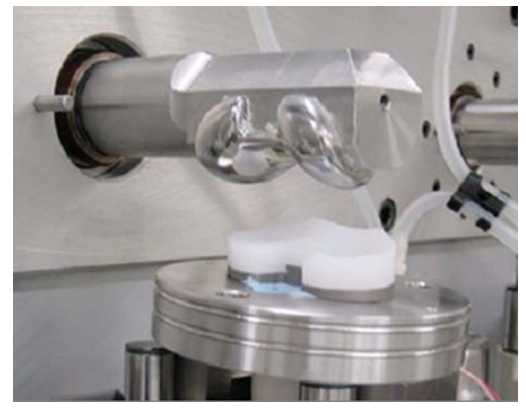

Fig. 1 Knee simulator test with Ti-6Al-4V alloy as the femoral component and the Hi-tech Knee II (Total Knee System, Nakashima Medical, Japan) with a flat surface, as the tibial component.

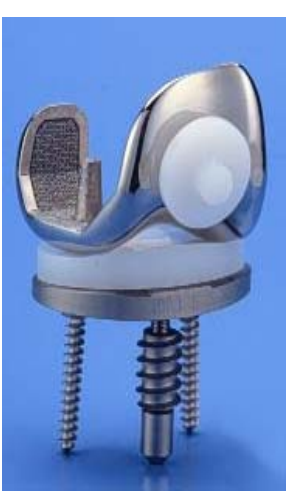

(a) Photograph.

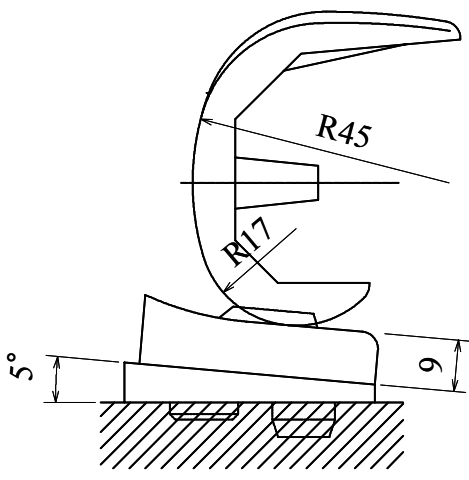

(b) Dimension.
Fig. 2 Component of Hi-tech Knee II Total Knee System (NAKASHIMA Medical, Japan). 
Isolation of UHMWPE wear debris from serum lubricant

UHMWPE wear debris was isolated from an aliquot of the serum lubricant used during 4.5 to 5 million cycles by incubation with potassium hydroxide $(85 \%$, KISHIDA REAGENTS CHEMICALS, Japan) at $60{ }^{\circ} \mathrm{C}$ for 48 hours in a water bath (PERSONAL-11, TAITEC, Japan) then chloroform (99\%, KISHIDA REAGENTS CHEMICALS, Japan) / methanol (99.8\%, KISHIDA REAGENTS CHEMICALS, Japan) (2:1 in v/v) was added to extract the lipids followed by centrifuging at $2000 \mathrm{G}$ at $4{ }^{\circ} \mathrm{C}$ for 10 minutes (Table top cooled centrifuges 2800, KUBOTA CORPARATION, Japan) 3 times. An equal volume of ethanol (99.5\%, KISHIDA REAGENTS CHEMICALS, Japan) was added, and any remaining contaminating proteins were precipitated with stirring at $4{ }^{\circ} \mathrm{C}$ for $24 \mathrm{~h}(\mathrm{HP} 41507$, International Scientific Instruments Supply Co., Ltd., Japan). After proteins were removed by centrifuging at $2000 \mathrm{G}$ at $4{ }^{\circ} \mathrm{C}$ for 2 hours, an equal volume of ultra pure water (arium611VF, sartorius, Germany) was added, and then sequentially filtered through a polycarbonate filter (Cyclopre ${ }^{\mathrm{TM}}$ Track Etched Membrane, Whatman International Ltd., UK)whose pore size was 10,1 and $0.1 \mu \mathrm{m}$ using a suction filtration system (SPC filter holder, SIBATA, JAPAN) equipped with a vacuum pump (Diaphragm Type Dry Vacuum Pump DAP-15, ULVAC KIKO Inc., Japan).

\section{Quantitative analysis of isolated UHMWPE wear debris}

Five random sections of the filter were cut after filtration and dried for 24 hours then coated with Pt (Ion Sputter, E-1030, HITACHI, Japan) followed by field emission scanning electron microscope (FE SEM, S-4500, HITACHI, Japan) observation. Images of the debris were observed using the FEGSEM analysed using ImageJ (free software downloaded from http://rsb.info.nih.gov/ij/, National Institute of Health, USA) to calculate the percentage and area of debris. Over 500 particles, which were identified undoubtedly as debris, for each sample were analysed from the randomly selected areas of the filters. The debris volume and volumetric concentration, $C(r)$, were calculated from following equation using the debris area in FEGSEM images, assuming a constant thickness.

$C(r)=\frac{\text { volume of debris in each size range }}{\text { volume of debris in all size range }} \times 100$

Form factor was calculated to evaluate debris shape by following equation.

Form factor $=4 \pi\left(\right.$ area $/$ perimeter $\left.^{2}\right)$.

A value of 1.0 indicates a perfect circle. As the value approaches 0 , it indicates an increasingly elongated shape.

All of the methods described above were the same as reported previously ${ }^{(1)}$.

Statistics

Throughout, the data was analysed by one-way ANOVA and the statistical significance was judged using the T-method to calculate minimum significant differences between group means. The data for non-oxidation accelerated Virgin UHMWPE and UHMWPE with VE which has been previously reported ${ }^{(1)}$ was plotted as reference in all results as well.

\section{Results}

As shown in Fig. 3, the total amount of wear volume was significantly different where the aged virgin UHMWPE had $534.6 \mathrm{~mm}^{3}$ and aged UHMWPE containing VE had 43.8 
$\mathrm{mm}^{3}$. The aged UHMWPE containing VE consistently showed more than a twelfth lower wear volume than that of the aged virgin UHMWPE and showed almost similar wear volume as non-aged virgin UHMWPE and UHMWPE with VE.

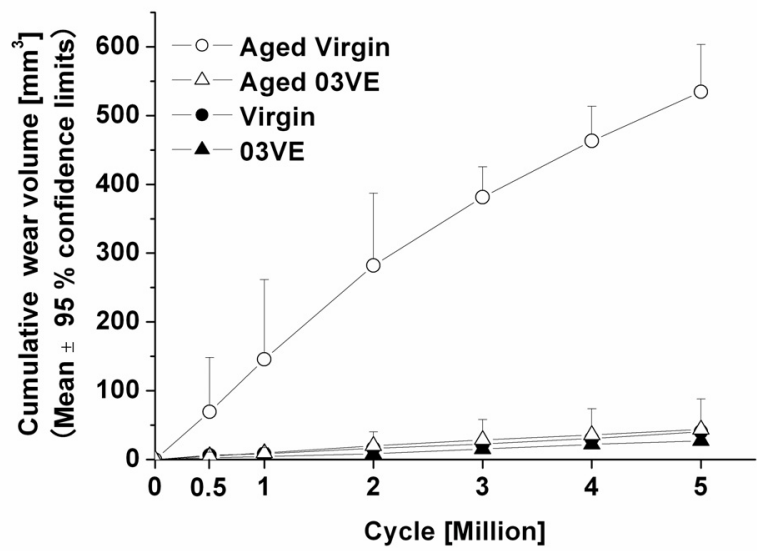

Figure. 3 Cumulative wear volume for each material calculated from the gravimetric measurement from 0.5 to 5 million cycles. Accelerated aging UHMWPE with VE consistently showed lower wear volume of more than a twelfth than that of the oxidation accelerated virgin UHMWPE and showed almost similar wear volume as non-oxidation accelerated virgin UHMWPE and UHMWPE with VE.

Images for typical debris of each sample in the smallest size range, between 0.1 and 1.0 $\mu \mathrm{m}$ were shown in Fig. 4. Various shape and size of debris was observed.

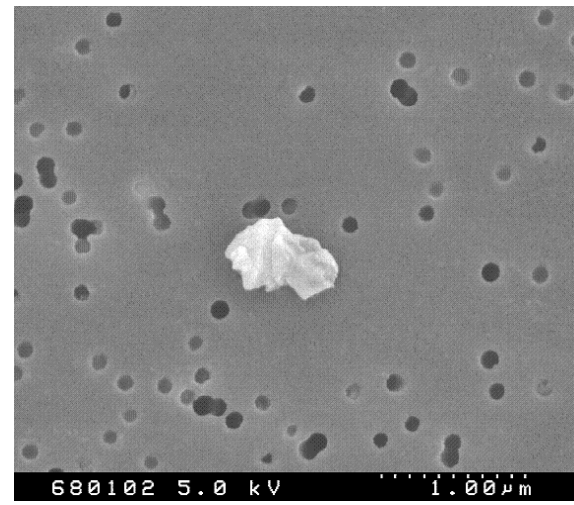

(a) Virgin UHMWPE

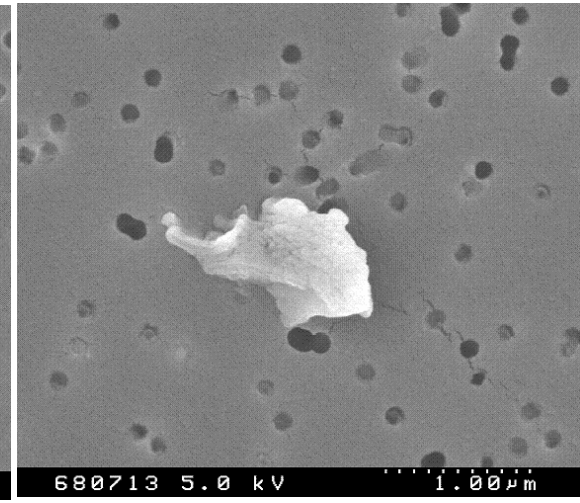

(b) UHMWPE containing VE

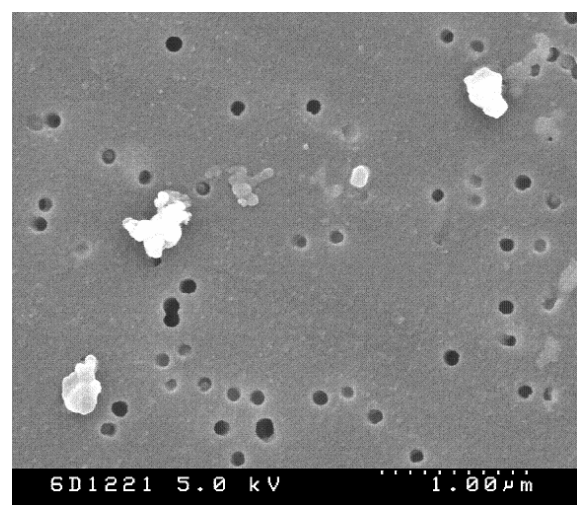

(c) Aged virgin UHMWPE

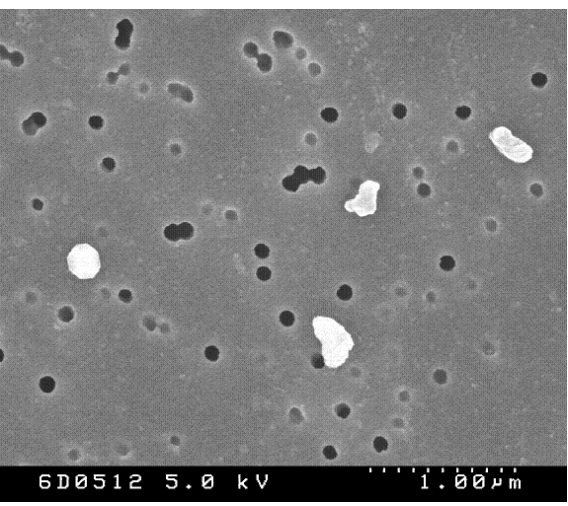

(d) Aged UHMWPE containing VE

Fig. 4 Images for typical debris of each sample. 
Volumetric concentration, $C(r)$, as a function of the longest length of debris is shown in Fig. 5. Although, there were no significant differences between all samples, the mode of the aged UHMWPE containing VE ranged from the smallest size in 0.1 to $1 \mu \mathrm{m}$.

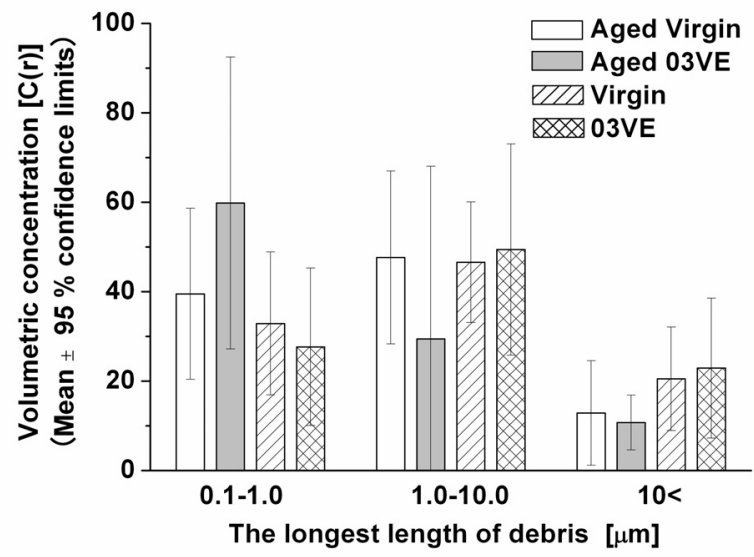

Figure. 5 Volumetric concentration, $\mathrm{C}(\mathrm{r})$, as a function of debris the longest length. Although, there were no significant differences between all samples, the mode of the aged UHMWPE with VE ranged from the smallest size in 0.1 to $1 \mu \mathrm{m}$.

There were no significant differences between all samples in the same size range (Fig. 6).

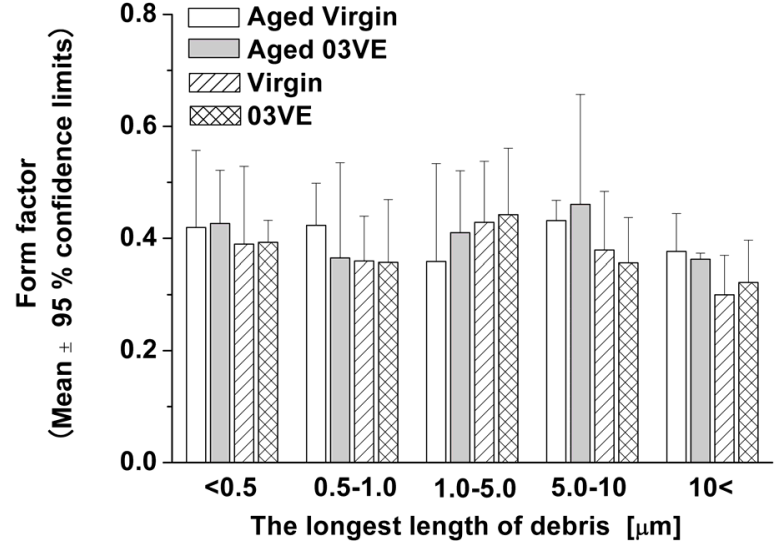

\section{Discussion}

Accelerated ageing treatment on UHMWPE has been proposed ${ }^{(17)-(19)}$ and standardised by the American Society for Testing and Materials (ASTM) ${ }^{(20)}$ to simulate ageing in a shelf for clinical use of UHMPWE. The aim of the current paper is not to simulate shelf ageing but to investigate the wear performance of the UHMWPE containing VE after the accelerated ageing treatment. Thus rather severe ageing treatment, $\gamma$-Irradiation and $80{ }^{\circ} \mathrm{C}$ for 23 days both in air condition were adopted in the current study although $\gamma$-Irradiation in air is not applied recently.

The current study showed wear reduction of aged UHMWPE by VE addition as shown in Fig. 3. In a comparison of non-aged and aged virgin UHMWPE, the ageing treatment made wear volume approximately twelvefold. Oxidation is very influential factor caused by the combination of free radicals with oxygen. Not only scission of molecular chain but also producing peroxides may occur by continuous oxidation process in UHMWPE.

One of the dominant effects of VE addition on wear reduction is thought to be the anti-oxidative properties of VE resulting in lower oxidative degradation in any situation ${ }^{(6)-(10)(15)}$. The continuous oxidation process may end due to lack of oxygen and/or as a result of the presence of relatively stable radicals, such as those of vitamin E. .However there is no 
direct evidence how vitamin E exists and work inside the UHMWPE.

In comparison to the wear volume of other studies, where almost similar conditions, kinematics for knee simulator and type of prosthesis but non ageing treatment was applied, the wear volume in the current study was approximately doubled ${ }^{(22)}$. This implies that the accelerated ageing treatment adopted in the current study approximately doubled the wear volume.

It is suggested that debris generated by wear from UHMWPE causes osteolysis ${ }^{(23)-(28)}$. It is recognised that sub-micron sized debris has especially higher reactive for cells to secrete cytokine inducing to osteolysis then finally resulting in aseptic loosening ${ }^{(26)(28)}$. The quantitative analysis of debris size distribution in Fig. 5 showed that the aged UHMWPE with VE had a higher percentage of sub-micron sized debris than the other three materials although there were not significant differences. And, on top of all, it has been reported that UHMWPE containing VE had reduced biological activity ${ }^{(29)}$. There is a potential that the biological activity is increased with higher oxidation levels in UHMWPE (30)-(32). Comprehensive evaluation including wear volume, wear debris shape, size distribution of wear debris and biological activity is required.

Regarding the debris shape, some researchers have advocated that the shape of UHMWPE wear debris may play an important role in adverse local tissue responses and elongated fibrils of the particles provoke a more severe inflammatory reaction by macrophage ${ }^{(33)}$. In Fig. 6 all of materials had no significant differences in debris shape. Thus, in terms of debris shape, UHMWPE with VE may have similar degree of influence on osteolysis.

It is hard to say how many years UHMWPE with VE must be fine after implantation due to limited techniques to simulate it precisely. However it can be described that UHMWPE with VE has shown lower wear volume and less influence on osteolysis than conventional UHMWPE even after rather severe oxidative environment according to the data in the current study.

\section{Conclusion}

Aged UHMWPE blended with VE showed lower wear volume than that of aged UHMWPE.

\section{Acknowledgement}

Part of this study was supported by risk taking fund for technology development, Japan science and technology agency. The authors would like to thank Mr. Sadamu Kinoshita for SEM observation.

\section{References}

(1)Teramura, S., Sakoda, H., Terao, T., Endo, M.M., Fujiwara, K., Tomita, N., Reduction of wear volume from ultrahigh molecular weight polyethylene knee components by the addition of vitamin E, Journal of Orthopaedic Research, Vol.26, No.4 (2008), pp.460-464.

(2)Oral, E., Wannomae, K.K., Hawkins, N., Harris, W.H., Muratoglu, O.K., Alpha-Tocopherol-doped irradiated UHMWPE for high fatigue resistance and low wear, Biomaterials, Vol.25, No.24 (2004), pp.5515-5522.

(3)Tomita, N., Kitakura, T., Onmori, N., Ikada, Y., Aoyama, E., Prevention of fatigue cracks in ultrahigh molecular weight polyethylene joint components by the addition of vitamin E, Journal of Biomedical Material Research, Vol.48, No.4 (1999), pp.474-478.

(4)Shibata, N., Tomita, N., Onmori, N., Kato, K., Ikeuchi, K., Defect initiation at subsurface grain boundary as a precursor of delamination in ultrahigh molecular weight polyethylene, Journal of Biomedical Material Research A, Vol.67, No.1 (2003), pp. 276-284.

(5)Wolf, C., Lederer, K., Bergmeister, H., Losert, U., Böck, P., Animal experiments with ultra-high molecular weight polyethylene (UHMW-PE) stabilised with alpha-tocopherol used for 
articulating surfaces in joint endoprostheses, Journal of Material Science Materials in Medicine, Vol.17, No.12 (2006), pp.1341-1347.

(6)Shibata, N., Tomita, N., The anti-oxidative properties of alpha-tocopherol in gamma-irradiated UHMWPE with respect to fatigue and oxidation resistance, Biomaterials, Vol.26, No.29 (2005), pp.5755-5562.

(7)Wolf, C., Krivec, T., Blassnig, J., Lederer, K., Schneider, W., Examination of the suitability of alpha-tocopherol as a stabilizer for ultra-high molecular weight polyethylene used for articulating surfaces in joint endoprostheses, Journal of Materials Science Materials in Medicine, Vol.13, No.2 (2002), pp.185-189.

(8)Oral, E., Greenbaum, E.S., Malhi, A.S., Harris, W.H., Muratoglu, O.K., Characterization of irradiated blends of alpha-tocopherol and UHMWPE, Biomaterials, Vol.26, No.33 (2005), pp. 6657-6663.

(9)Mori, A., Tomita, N., Kaneeda, T., Nagata, K., Moriya, H., Osaka, A., Kuramoto, K., Wear resistance of UHMWPE mixed vitamin E supposing lift-off in TKA, Proceedings of 48th Annual Meeting of Orthopaedic Research Society, (2002), pp.1041.

(10)Wolf, C., Macho, C., Lederer, K,. Accelerated ageing experiments with crosslinked and conventional ultra-high molecular weight polyethylene (UHMW-PE) stabilized with alpha-tocopherol for total joint arthroplasty, Journal of Materials Science Materials in Medicine, Vol.17, No.12 (2006), pp.1333-1340.

(11)Wolf, C., Lederer, K., Pfragner, R., Schauenstein, K., Ingolic, E., Siegl, V., Biocompatibility of ultra-high molecular weight polyethylene (UHMW-PE) stabilized with alpha-tocopherol used for joint endoprostheses assessed in vitro, Journal of Materials Science Materials in Medicine, Vol.18, No.6 (2007), pp.1247-1252.

(12)Renò, F., Cannas, M., UHMWPE and vitamin E bioactivity: An emerging perspective, Biomaterials, Vol.27, No.16, (2006), pp.3039-3043.

(13)Oral, E., Godleski, B.C., Malhi, A.S., Muratoglu, O.K., The effects of high dose irradiation on the cross-linking of vitamin E-blended ultrahigh molecular weight polyethylene, Biomaterials, Vol.29, No.26 (2008), pp.3557-3560.

(14)Parth, M., Aust, N., Lederer, K., Studies on the effect of electron beam radiation on the molecular structure of ultra-high molecular weight polyethylene under the influence of alpha-tocopherol with respect to its application in medical implants, Journal of Materials Science Materials in Medicine, Vol.13, No.10 (2002), pp.917-921.

(15)Oral, E., Rowell, S.L., Muratoglu, O.K., The effect of alpha-tocopherol on the oxidation and free radical decay in irradiated UHMWPE, Biomaterials, Vol.27, No.32 (2006), pp.5580-5587.

(16)Oral, E., Christensen, S.D., Malhi, A.S., Wannomae, K.K., Muratoglu, O.K., Wear resistance and mechanical properties of highly cross-linked, ultrahigh-molecular weight polyethylene doped with vitamin E, Journal of Arthroplasty, Vol.21, No.4 (2006), pp.580-591.

(17)Sun, D.C., Scmidig, G., Stark, C., A simple accelerated aging method for simulations of long-term oxidative aging effects in UHMWPE implants, Proceedings of 42nd Annual meeting of Orthopaedic Research Society, (1996), pp.493.

(18)Sanford, W.M., Saum, K.A., Accelerated oxidative aging testing of UHMWPE, Proceedings of 41st Annual meeting of Orthopaedic Research Society, (1995), pp.119.

(19)Kurtz, S.M., Muratoglu, O.K., Evans, M., Edidin, A.A., Advances in the processing, sterilization, and crosslinking of ultra-high molecular weight polyethylene for total joint arthroplasty, Biomaterials, Vo.20, No.18 (1999), pp.1659-1688.

(20)American Society for Testing and Materials. Standard guide for accelerated aging of ultra high molecular weight polyethylene, ASTM F 2003-00, (2003).

(21)Sakoda, H., Fisher, J., Lu, S., Buchanan, F., The effect of accelerated aging on the wear of UHMWPE, Journal of Materials Science Materials in Medicine, Vol.12, No.10-12 (2001), pp.1043-1047.

(22)McEwen, H.M., Barnett, P.I., Bell, C.J., Farrar, R., Auger, D.D., Stone, M.H., Fisher, J., The 
influence of design, materials and kinematics on the in vitro wear of total knee replacements, Journal of Biomechanics, Vol.38, No.2 (2005), pp.357-365.

(23)Revell, P.A., Al-Saffar, N., Kobayashi, A., Biological reaction to debris in relation to joint prostheses, Proceedings of the Institution of Mechanical Engineers. Part H, Journal of engineering in medicine, Vol.211, No.2 (1997), pp.187-197.

(24)Schmalzried, T.P., Jasty. M., Harris, W.H., Periprosthetic bone loss in total hip arthroplasty: polyethylene wear debris and the concept of the effective joint space, Journal of Bone and Joint Surgery American volume, Vol.74, No.6, (1992), pp.849-863.

(25)Amstutz, H.C., Campbell, P., Kossovsky, N., Clarke, I.C., Mechanism and clinical significance of wear debris-induced osteolysis, Clinical Orthopaedic Related Research, Vol.276, (1992), pp.7-18.

(26)Ingham, E., Fisher, J., Biological reactions to wear debris in total joint replacement, Proceedings of the Institution of Mechanical Engineers, Part H, Journal of engineering in medicine, Vol.214, No.1 (2000), pp.21-37.

(27)Savio, J.A., Overcamp, L.M., Black, J., Size and shape of biomaterial wear debris, Clinical Materials,Vol.15, No.2 (1994), pp.101-147.

(28)Green, T.R., Fisher, J., Stone, M., Wroblewski, B.M., Ingham, E., Polyethylene particles of a "critical size" are necessary for the induction of cytokines by macrophages in vitro, Biomaterials, Vol.19, No.24 (1998), pp.2297-2302.

(29)Teramura, S., Russell, S., Ingham, E., Fisher, J., Tomita, N., Fujiwara, K., Tipper, J.L., Reduced biological response to wear particles from UHMWPE containing vitamin E, Proceedings of 55th Annual Meeting of Orthopaedic Research Society, (2009), pp.2377.

(30)Brach del Prever, E.M., Bistolfi, A., Costa, L., Bracco, P., Linari, A., Botto, M.F., Crova, M., Gallinaro, P., The biological reaction to polyethylene wear debris can be related with oxidation of the UHMWPE cups. La Chirurgia degli organi di movimento, Vol.88, No.3 (2003), pp.291-303.

(31)Bosetti, M., Zanardi, L., Bracco, P., Costa, L., Cannas, M., In vitro evaluation of the inflammatory activity of ultra-high molecular weight polyethylene, Biomaterials, Vol.24, No. 8 (2003), pp.1419-1426.

(32)Renò, F., Cannas, M., Costa, L., UHMWPE oxidation increases matrix metalloproteinase-2 (MMP-2) release in human fibroblast, Journal of Materials Science Materials in Medicine, Vol.13, No.4 (2002), pp.403-406.

(33)Ren, W., Yang, S.Y., Fang, H.W., Hsu, S., Wooley, P.H., Distinct gene expression of receptor activator of nuclear factor-kappaB and rank ligand in the inflammatory response to variant morphologies of UHMWPE particles, Biomaterial, Vol.24, No.26 (2003), pp.4819-4826. 